\title{
THE BENEFITS OF TERRESTRIAL LASER SCANNING AND HYPERSPECTRAL DATA FUSION PRODUCTS
}

\author{
S. J. Buckley ${ }^{\mathrm{a},}{ }^{*}$, T. H. Kurz ${ }^{\mathrm{a}}$, D. Schneider ${ }^{\mathrm{b}}$ \\ ${ }^{a}$ Uni CIPR, Postboks 7810, N-5020 Bergen, Norway - simon.buckley@uni.no, tobias.kurz@uni.no \\ ${ }^{\mathrm{b}}$ Technische Universität Dresden, Institute of Photogrammetry and Remote Sensing, D-01062 Dresden, Germany - \\ danilo.schneider@tu-dresden.de
}

TS VII/3, VII/6, III/2, V/3: Integration of hyperspectral and lidar data

KEY WORDS: Hyper spectral, lidar, fusion, visualization, analysis, texture, value-added, photo-realism

\begin{abstract}
:
Close range hyperspectral imaging is a developing method for the analysis and identification of material composition in many applications, such as in within the earth sciences. Using compact imaging devices in the field allows near-vertical topography to be imaged, thus bypassing the key limitations of viewing angle and resolution that preclude the use of airborne and spaceborne platforms. Terrestrial laser scanning allows 3D topography to be captured with high precision and spatial resolution. The combination of 3D geometry from laser scanning, and material properties from hyperspectral imaging allows new fusion products to be created, adding new information for solving application problems. This paper highlights the advantages of terrestrial lidar and hyperspectral integration, focussing on the qualitative and quantitative aspects, with examples from a geological field application. Accurate co-registration of the two data types is required. This allows $2 \mathrm{D}$ pixels to be linked to the 3D lidar geometry, giving increased quantitative analysis as classified material vectors are projected to 3D space for calculation of areas and examination of spatial relationships. User interpretation of hyperspectral results in a spatially-meaningful manner is facilitated using visual methods that combine the geometric and mineralogical products in a 3D environment. Point cloud classification and the use of photorealistic modelling enhance qualitative validation and interpretation, and allow image registration accuracy to be checked. A method for texture mapping of lidar meshes with multiple image textures, both conventional digital photos and hyperspectral results, is described. The integration of terrestrial laser scanning and hyperspectral imaging is a valuable means of providing new analysis methods, suitable for many applications requiring linked geometric and chemical information.
\end{abstract}

\section{INTRODUCTION}

Applications in photogrammetry and remote sensing are often enhanced by the use of multiple sensors that provide complementary information and therefore improved products for users. A common example is the combination of laser scanning and photogrammetry, both terrestrial and aerial, where the high density and precise geometric data from lidar is given added value using the increased radiometric, spectral and spatial resolution provided by digital metric or semimetric imagery (e.g. Axelsson, 1999; Frueh et al., 2005; Fassi et al., 2011; Guo et al., 2011). Multispectral or hyperspectral sensors have the potential to further complement laser scanning topographic data, as an extended part of the electromagnetic spectrum is recorded with a greater number of spectral bands. Such sensors measure many narrow bands, allowing near-continuous reflectance curves to be derived per component image pixel (van der Meer and de Jong, 2001). Such high spectral sampling makes it possible to analyse small variations in material composition, even at the subpixel range (Keshava and Mustard, 2002). While the value of combined airborne lidar and hyperspectral data, for example in forestry, is being realised (Koch, 2010), in close range applications the integration is only now emerging as a potential means of linking geometrical and material properties (Kurz et al., 2008).

\footnotetext{
* Corresponding author.
}

Compact and portable hyperspectral sensors are now available, and are applicable to many close range applications, especially in the earth sciences, where the remote analysis of mineralogical distribution is often important. Such sensors have high spectral resolution, allowing increasingly complex mineral variations to be detected (Kurz et al., 2012). In addition, the close range instrument position makes it possible to image steep terrain and building façades, without the traditional problems associated with airborne and spaceborne imaging where spatial resolution and a nadir field of view are problematic. The integration of hyperspectral imagery with terrestrial laser scanning (TLS) has high potential for assisting application experts with interpretation and validation of classification results, owing to the improved spatial component provided by linking 2D spectral data to 3D lidar geometry.

This paper assesses the benefits of integrating TLS data and close range hyperspectral imagery, focussing on the combined products obtainable from these complementary techniques. A geological application is used to illustrate the proposed data fusion products. The paper is structured to give a background to the component techniques and the multi-sensor integration (Section 2), before the data collection, processing and registration is described (Section $3)$. Section 4 outlines visual methods for exploring the combined data, whilst Section 5 covers quantitative methods and accuracy assessment. 


\section{BACKGROUND}

TLS has become an established method for obtaining 3D geometry in many applications, such as architecture, mining, geology, and the wider earth sciences. In these disciplines, the method has become attractive, due to the rapid data acquisition, the high point precision and the ease of use, which has resulted in the technique being adopted by domain experts outside the geomatics field. Because laser scanning is an active technique, the strength of the returned laser pulse, commonly referred to as intensity or amplitude, is recorded (Höfle and Pfeifer, 2007). Intensity gives some benefit for object discrimination, as it is based on the absorption and reflectance properties of the measured material at the wavelength of the laser beam used (Bellian et al., 2005; Franceschi et al., 2009; Nield et al., 2011). However, as the laser operates within a very narrow spectral range, restricted to a single band, often in the near-infrared, the intensity values may only be practical for differentiating major material differences (Kurz et al., 2011).

To obtain a greater spectral resolution, a number of studies have used multispectral approaches. Lichti (2005) classified laser scanned scenes using the true colour (red, green, blue: RGB) channel and single infrared band of the used instrument. Hemmleb et al. (2006) developed a multispectral laser scanner, by combining several laser sources at different wavelengths, for studying building damage. Most recently close range hyperspectral sensors have been used to separate a wider range of materials, such as spectrally similar rock types (Kurz et al., 2012).

It is common for digital imagery to be collected simultaneously with laser scans. A number of instruments integrate a digital camera into the acquisition pipeline, giving access to calibrated semi-metric imagery of high resolution (e.g. Buckley et al., 2008). Though a point cloud may be displayed using the intensity value for colour, the inherently sparse, or discontinuous, data can make detailed interpretation difficult. Features may have a minimal trace in $3 \mathrm{D}$, but may be obvious in $2 \mathrm{D}$ images as colour or edges. For this reason, photorealistic modelling, combining surface data and imagery, is a standard approach. Converting a lidar point cloud to a triangular mesh makes the surface representation continuous, and colour information from digital photos can be texture mapped to create an integrated product (El-Hakim et al., 1998; Frueh et al., 2005). Such a photorealistic model offers value for visualisation, education and quantitative measurement of recorded objects (e.g. Bellian et al., 2005).

Despite the advantages of combining terrestrial laser and image data in photorealistic models, little quantitative information is provided on the mineral and chemical composition of the scanned object. This lack provides the motivation for integrating a further data type, close range hyperspectral imagery, into the modelling workflow, with the associated benefits of an extended spectral range and resolution.

\section{DATASET AND PROCESSING WORKFLOW}

\subsection{Application}

Geology is one application field where terrestrial laser scanning integrated with conventional visible light digital imaging has become widespread (Bellian et al., 2005; Buckley et al., 2008; Buckley et al., 2010). Photorealistic models are created of geological outcrops, allowing both surface topography and geological features to be studied in detail. Because well-exposed outcrops often comprise high and steep cliff sections and quarry faces, non-contact measurement techniques are essential. While mineralogical study has been possible in the past using limited spot samples, close range hyperspectral imaging offers a remote and high resolution means of analysing chemical distribution over larger areas. Example data from geological outcrop studies are used here to illustrate the potential benefits of combined TLS and hyperspectral scanning.

\subsection{Instrumentation and data collection}

Data were collected from two sites, the first a cliff section at Garley Canyon, Utah, USA, the second a quarry wall at Pozalagua, Cantabria, Spain (Fig. 1). A Riegl LMS-Z420i terrestrial laser scanner was used to collect point clouds of the two outcrops, and a calibrated Nikon D200 camera with a Nikkor $85 \mathrm{~mm}$ lens, mounted on top of the scanner, provided imagery registered in the lidar coordinate system. Several scans were collected per outcrop to avoid shadows caused by obstruction from the individual instrument positions (two for Garley Canyon and seven at Pozalagua). A global navigation satellite system (GNSS) was used to record the position of each scan position (Buckley et al., 2010).

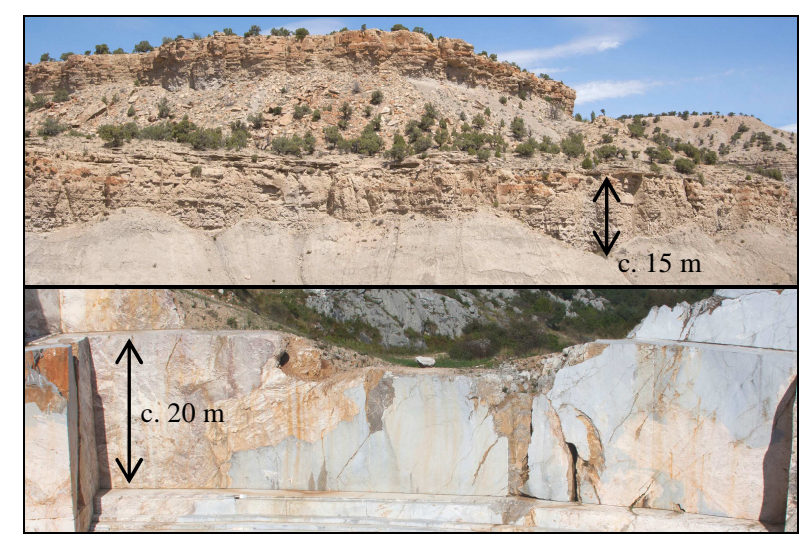

Figure 1. Outcrops at Garley Canyon (top) and the Pozalagua Quarry (bottom).

A HySpex SWIR-320m hyperspectral imager (Norsk Elektro Optikk, 2012) was used to image each of the two outcrops. The HySpex imager records 240 bands across the short-wave infrared (SWIR) part of the electromagnetic spectrum, with a spectral sampling of $5 \mathrm{~nm}$. The spatial dimension of the sensor contains 320 pixels, effectively resulting in line (pushbroom) scanning when used with a rotation stage. Because rotation is required to build up the number of image columns, the width of an image is variable, determined by the user. This configuration results in panoramic imaging geometry, where only the across-track image direction can be represented by central perspective projection (Luhmann et al., 2006; Schneider and Maas, 2006). Spatial resolution is low compared with a contemporary digital camera, but the high spectral resolution allows greater differentiation of mineral content. In this study, multiple images were collected for each area, and one representative image was selected for hyperspectral processing and data fusion for both sites. 
To facilitate co-registration of the hyperspectral and lidar data, a number of retro-reflective targets were placed in accessible parts of the outcrop. These were recorded by both systems, and used later as control points for hyperspectral image registration. Spectral calibration targets were additionally located on the outcrop and imaged using the HySpex sensor.

\subsection{Processing}

For the TLS and hyperspectral fusion products, the requirements are that the registration of each dataset is performed in the same coordinate system. Laser scans must be registered, to a global or national system if required, and hyperspectral images must be registered to the laser data.

The laser scans were registered using surface matching and then transformed to global coordinates using the GNSS measurements. The point clouds were merged and reduced in areas of high overlap. A mesh was fit to the area of geological interest, and finally texture mapping was performed to create a photorealistic model of each outcrop using the conventional digital images. Full details are in Buckley et al. (2010).

Previous work (Kurz et al., 2011) has shown that the geometric model for the HySpex sensor can be represented by a standard model for linear-array panoramic cameras (Schneider and Maas, 2006). Additional parameters defined in this model account for the horizontal pixel size defined by the rotation step, and the non-parallelism of the sensor line and rotation axis. 3D control points were defined using the retro-reflective targets in the scan data, as well as by manually measuring natural control points to increase redundancy and improve the distribution of points in the hyperspectral imagery. Conjugate points were located in the hyperspectral radiance images. Finally, bundle adjustment was used to obtain the exterior orientation parameters (EOPs) of each hyperspectral image, along with an estimation of the camera calibration parameters (Kurz et al., 2011).

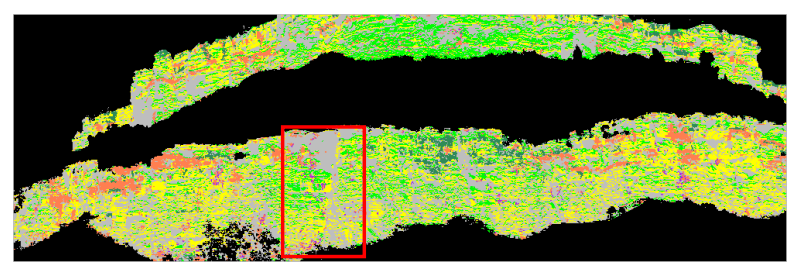

Figure 2. Classification of Garley Canyon outcrop (Light green: siltstone; yellow: sandstone; orange: carbonate; dark green: weathering; purple: gypsum; grey: unclassified; black: masked). Rectangle shows location of Fig. 4.

The full workflow for hyperspectral processing is presented in Kurz et al. (2012) and is only briefly summarised here. Sensor calibration was used to transfer the raw image measurements to reflectance, and artefacts pertaining to bad pixels, striping and brightness gradients were removed during pre-processing. The Maximum Noise Fraction transform (MNF; Green et al., 1988) was applied to reduce image noise and extract the salient spectral features from the high dimensional hyperspectral data cube. Classification was performed based on the mixture-tuned matched filtering
(MTMF) approach (Boardman, 1998), using end-members defined in the outcrop (e.g. Fig. 2).

\section{VISUAL DATA FUSION METHODS}

Following successful registration, hyperspectral images and classification products can be used in a spatially-meaningful manner, both qualitatively and quantitatively. This section presents possibilities for visual combination of hyperspectral results with TLS data.

During registration, the reflectance images are used for measuring control points. However, hyperspectral processing results in many image products, from multiple MNF band combinations to classification output from different algorithms (Fig. 3). As long as the pixel dimensions are not changed from that of the acquired image, the same EOPs can be used for any number of processing products.

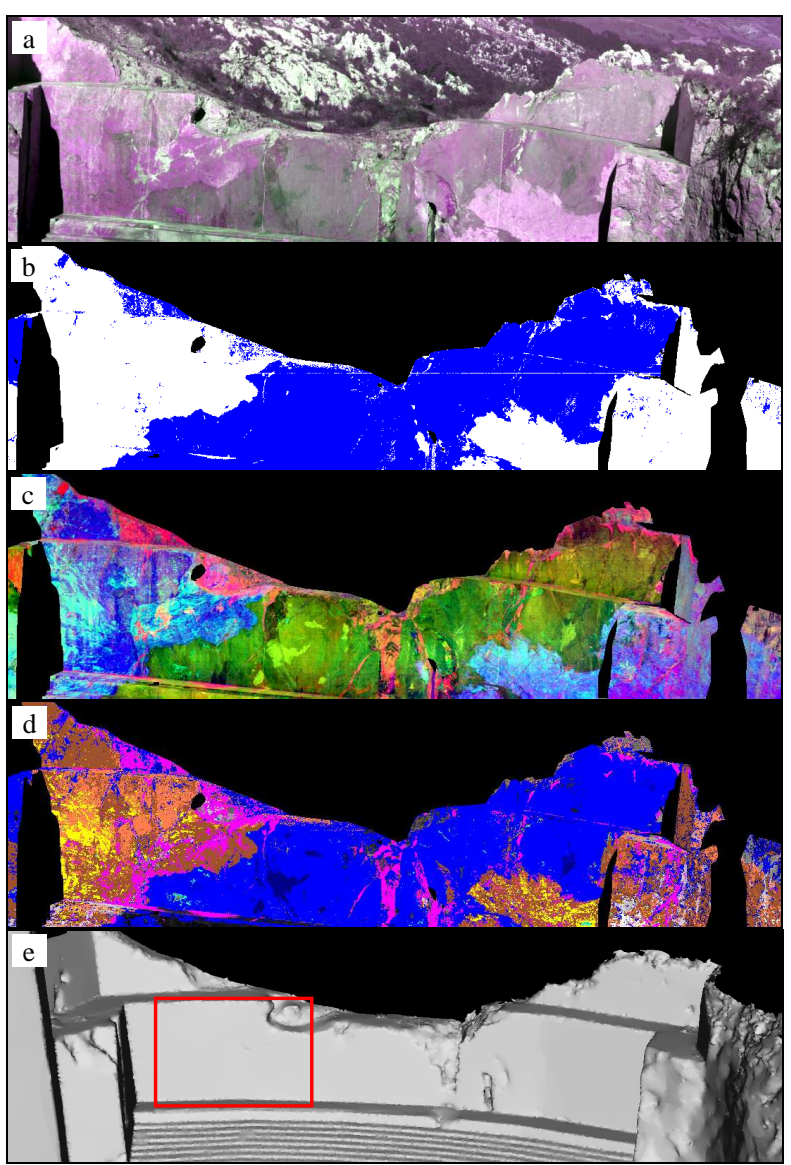

Figure 3. Hyperspectral processing results for one image of the Pozalagua Quarry. a) radiance image; b) band ratio image showing limestone (blue) and dolomite (white); c) MNF image with three bands shown in false colour; d) MTMF classification of outcrop materials; e) corresponding lidar geometry of quarry face. Rectangle shows location of Fig. 5.

\subsection{Coloured point clouds and meshes}

A simple method of combining hyperspectral results with the lidar geometry is by colouring the point cloud, in the same way as is often carried out with conventional digital images 
in laser scanning software. The colouring is performed using the EOPs of the image, the geometric model for the sensor and the collinearity condition to obtain $2 \mathrm{D}$ pixel coordinates for each 3D lidar point. The 2D position is used to assign RGB values to the $3 \mathrm{D}$ points (Fig 4a). Advantages of the method are that a 3D impression of the material distribution can be quickly created, and points not in the geologically relevant parts of the scene can be rapidly highlighted and removed. The point cloud can easily be classified and segmented into the material classes. However, disadvantages are that point clouds are discontinuous (as described in Section 2), and during colouring no account is taken for geometric obstructions in the camera's field of view between a given point and the image plane, making incorrect colouring a possibility when images are not captured from a similar position as the laser scanner. These disadvantages can be alleviated using triangle meshes rather than point clouds in that case the surface model is continuous, and more occluding geometry can be used to test visibility when colouring the mesh vertices (Fig 4b).

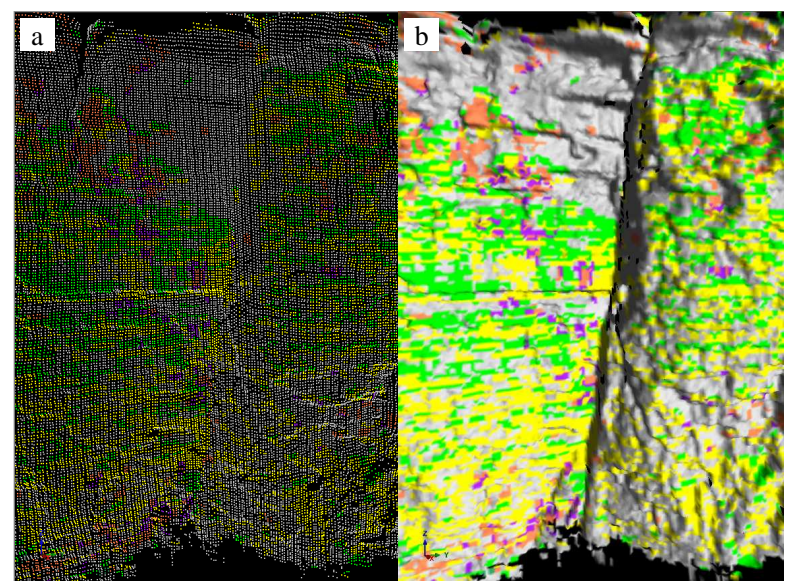

Figure 4. Lidar geometry coloured using classification image in Fig. 2. a) point cloud; b) mesh. Height of area c. $15 \mathrm{~m}$.

\subsection{Photorealistic modelling with multiple textures}

A mesh makes it possible to visualise the coloured lidar geometry in a continuous form, aiding ease of interpretation. However, the quality of the result is a function of the number of vertices and triangles in the mesh, where large triangles will cause degradation in detail. Texture mapping relates image pixels to the mesh geometry, making use of the full image resolution independently of mesh detail. During texture mapping, the vertices of each mesh triangle are projected into an appropriate image, defining an area of pixels to be used as texture. All defined patches are saved to one or more texture images and the position of each patch is stored as a property of the vertices in the original mesh. In $3 \mathrm{D}$ viewing software, the mesh and texture images are loaded, and the stored texture coordinates define the linkage between vertices and image data, allowing on-screen rendering of the photorealistic model.

Hyperspectral classifications can be textured on the lidar model; however, the value is limited by the coarse resolution of the HySpex sensor. Superimposing hyperspectral results on an existing photorealistic model (lidar geometry textured with conventional digital images) provides a far more useful application of texture mapping. This multi-layer approach gives greater context to the thematic maps and is valuable for interpretation and validation.

Software for performing the multi-layer texture mapping was implemented, along with 3D viewing software for interactively manipulating the layer combinations. Input data are the mesh model, Nikon imagery, and multiple hyperspectral images and processing products. In addition, a project file stores EOPs of all images as well as a designated layer number that each image should be mapped to. A typical layer configuration is for conventional images to form the first layer, and then hyperspectral results, such as MNF images and MTMF classifications to form subsequent layers.

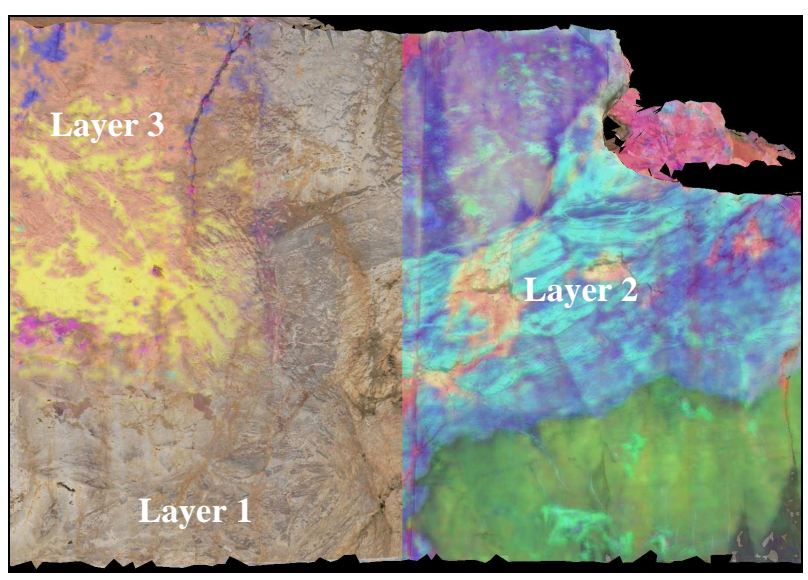

Figure 5. Photorealistic model combining conventional digital imagery and multiple hyperspectral processing layers. Layer 1 is the Nikon imagery ( $0 \%$ transparency). Layer 2 is an MNF image (Fig. 2c), with 50\% transparency, 50\% horizontal cutoff and a sharp edge transition. Layer 3 is a classification (Fig. 2d), with $40 \%$ transparency, a horizontal and vertical cutoff, and a soft edge transition. Height of model c. $20 \mathrm{~m}$.

The viewing software was written in $\mathrm{C}++$ using the OpenSceneGraph library for 3D rendering, providing a highlevel interface to OpenGL. OpenGL supports the use of multiple texture units, termed multitexturing, defining how image textures are stored and used in the graphics system. The number of layers is restricted only by the number of texture units supported by the graphics card of the computer viewing the processed model. During rendering, the layer combination is controlled using OpenGL state properties, making it possible to set the contribution of each texture unit. Most modern graphics cards allow the use of programmable parts of the graphics pipeline. The OpenGL Shading Language offers a high amount of flexibility for multiple textures, as user-defined functions for texture combination can be written and sent to the graphics card as fragment shaders. To take advantage of this, each layer is additionally given a transparency factor (0 to $100 \%$ ) specifying the overall weighting of the layer. Horizontal and vertical factors are also given, to allow side-by-side visualisation of multiple layers. Each parameter is linked to a slide control in the graphical user interface, allowing interactive adjustment of the layer weighting (Fig. 5). The horizontal and vertical edge transition can be feathered to give hard or soft edges. 


\section{GEOMETRIC DATA FUSION METHODS}

\subsection{Classification vectors}

Classification algorithms result in the assignment of pixels to different material classes, which are represented by 2D image space vectors in remote sensing software. Using the EOPs of the hyperspectral image, the reverse collinearity condition can be used to form rays for each point of a $2 \mathrm{D}$ line. As the image has no depth information, the lidar mesh is intersected by the rays to give points in object space, thus transforming the classification boundaries into 3D polylines and polygons. This 3D information can be used to extract real-world dimensions, allowing investigation of spatial relationships of material distribution.

\subsection{Point cloud classification and modelling}

The 3D classification boundaries obtained in Section 5.1 can be combined with segmented point clouds (Section 4.1) to create triangulated meshes for individual material bodies. This allows accurate calculation of surface area for the bodies, and estimation of volumes where appropriate 3D exposure is present. The procedure operates on a single material class at a time; i.e. a set of lines and a segmented point cloud representing one class. Each line is processed in turn, first checking whether it is the outer boundary of a body, or whether it is an inner hole indicative of a different material class. All points of the point cloud are projected to image space using the point of view of the capturing image, to test whether they are inside the polygon. Polygons with no containing points are designated as holes. Still working in image space, the points and lines are triangulated, using the holes as constraints for triangle removal. Finally, the internal vertices of the triangulated body are assigned their original 3D lidar positions, and the points on the line segments are projected to 3D as in Section 5.1 (Fig. 6).

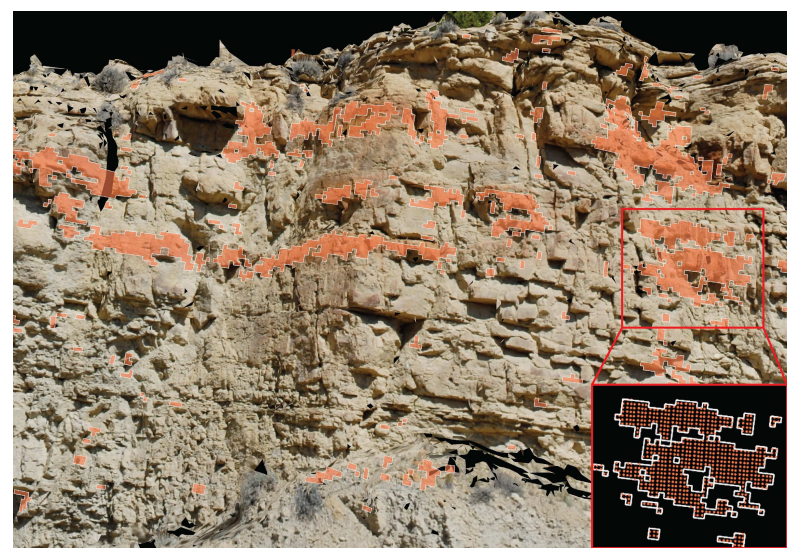

Figure 6. 3D material bodies created using triangulated points and classification boundaries, and superimposed on textured model (Garley Canyon). Inset shows detail of points and lines.

\subsection{Assignment of geometric properties to imagery}

Using the TLS geometry allows hyperspectral image information to be accessed in $3 \mathrm{D}$ in object space units. For remote sensing processing it can be useful to have per-pixel geometric information, such as for topographic correction and spatial analysis of results. For each image pixel, a ray is defined and intersected with the 3D mesh, as in Section 5.1.
For pixels with no 3D data, such as those representing sky or background, a no-data value is assigned. The surface normal at the intersection point can be used to calculate slope and aspect values for each pixel (e.g. Fig. 7), which are useful for topographic correction and geological analysis. In addition, the range from the camera centre to the intersected points can be used for calculating object sample distance, giving a quick approximation for material class areas in the remote sensing software environment.

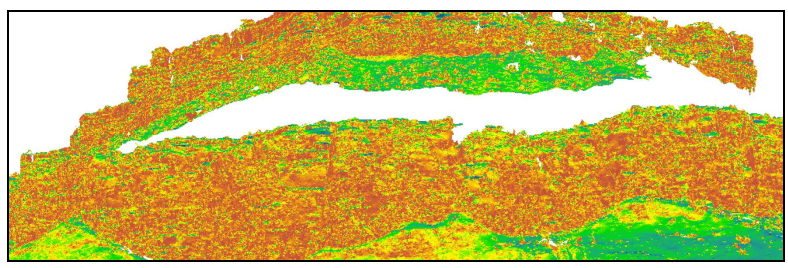

Figure 7. Slope-encoded image that can be used as a processing mask in remote sensing software. White areas are outside of the 3D model. Garley Canyon image used (Fig. 2).

\subsection{Accuracy considerations}

Multi-sensor fusion products rely on accurate co-registration of the component techniques to be used appropriately. For close range hyperspectral data, the image registration is critical and should be inspected. Whilst statistical confidence is a result of bundle adjustment, it is also important to evaluate the accuracy in an integrated way. Photorealistic models (Section 4.2) textured with conventional photos and a hyperspectral product give a qualitative indication of registration accuracy, as the user can blend between the layers to see the proximity of conjugate areas in both data types. Projection of the classification vectors to 3D allows their position with respect to the photorealistic model to be compared geometrically (Kurz et al., 2011).

\section{CONCLUSIONS}

This paper has outlined the potential of integrated TLS and close range hyperspectral data for simultaneously analysing the geometry and distribution of materials. Examples from an application in geology have been used to illustrate the data fusion products, though the methods have potential use in many other disciplines. The two data types are highly complementary, and should be used together both during processing and for later analysis. Results are often visual, and it may be a challenge to link all information in a single environment for interpretation. Photorealistic modelling with multiple textures allows the hyperspectral results to be spatially related to higher resolution conventional photos, and lidar geometry, in a single interactive viewing framework. Linking 2D image data to $3 \mathrm{D}$ introduces quantitative means for analysing classification results, and allows co-registration accuracy to be inspected both statistically and visually.

\section{ACKNOWLEDGEMENTS}

This work was supported in part by the Research Council of Norway's Petromaks programme (grants 163264 and 176132). Statoil ASA is thanked for supporting fieldwork at 
the Pozalagua Quarry, and for a travel grant allowing presentation of this paper. Norsk Elektro Optikk AS and Riegl $\mathrm{GmbH}$ are acknowledged for continued hardware and software support. John Howell is thanked for assistance with fieldwork in Spain.

\section{REFERENCES}

Axelsson, P., 1999. Processing of laser scanner data algorithms and applications. ISPRS Journal of Photogrammetry and Remote Sensing, 54(2-3), pp. 138-147.

Bellian, J.A., Kerans, C. and Jennette, D.C., 2005. Digital outcrop models: applications of terrestrial scanning lidar technology in stratigraphic modeling. Journal of Sedimentary Research, 75(2), pp. 166-176.

Boardman, J.W., 1998. Leveraging the high dimensionality of AVIRIS data for improved sub-pixel target unmixing and rejection of false positives: mixture tuned matched filtering. Summaries of the Seventh Annual JPL Airborne Earth Science Workshop, Pasadena, California. Vol. 1, pp. 55.

Buckley, S.J., Howell, J.A., Enge, H.D. and Kurz, T.H., 2008. Terrestrial laser scanning in geology: data acquisition, processing and accuracy considerations. Journal of the Geological Society, London, 165(3), pp. 625-638.

Buckley, S.J., Enge, H.D., Carlsson, C. and Howell, J.A., 2010. Terrestrial laser scanning for use in virtual outcrop geology. Photogrammetric Record, 25(131), pp. 225-239.

El-Hakim, S.F., Brenner, C. and Roth, G., 1998. A multisensor approach to creating virtual environments. ISPRS Journal of Photogrammetry and Remote Sensing, 53(6), pp. 379-391.

Fassi, F., Achille, C. and Fregonese, L., 2011. Surveying and modelling the main spire of Milan Cathedral using multiple data sources. Photogrammetric Record, 26(136), pp. 462487.

Franceschi, M., Teza, G., Preto, N., Pesci, A., Galgaro, A. and Girardi, S., 2009. Discrimination between marls and limestones using intensity data from terrestrial laser scanner. ISPRS Journal of Photogrammetry and Remote Sensing, 64(6), pp. 522-528.

Frueh, C., Jain, S. and Zakhor, A., 2005. Data processing algorithms for generating textured $3 \mathrm{D}$ building facade meshes from laser scans and camera images. International Journal of Computer Vision, 61(2), pp. 159-184.

Green, A.A., Berman, M., Switzer, P. and Craig, M.D., 1988. A transformation for ordering multispectral data in terms of image quality with implications for noise removal. IEEE Transactions on Geoscience and Remote Sensing, 26(1), pp. 65-74.

Guo, L., Chehata, N., Mallet, C. and Boukir, S., 2011. Relevance of airborne lidar and multispectral image data for urban scene classification using Random Forests. ISPRS Journal of Photogrammetry and Remote Sensing, 66(1), pp. 56-66.

Hemmleb, M., Weritz, F., Schiemenz, A., Grote, A. and Maierhofer, C., 2006. Multi-spectral data acquisition and processing techniques for damage detection on building surfaces. International Archives of the Photogrammetry, Remote Sensing and Spatial Information Sciences, Vol. XXXVI, Part B5, 6 pages (on CD-ROM).

Höfle, B. and Pfeifer, N., 2007. Correction of laser scanning intensity data: data and model-driven approaches. ISPRS Journal of Photogrammetry and Remote Sensing, 62(6), pp. 415-433.

Keshava, N. and Mustard, J.F., 2002. Spectral unmixing. IEEE Signal Processing Magazine, 19(1), pp. 44-57.

Koch, B., 2010. Status and future of laser scanning, synthetic aperture radar and hyperspectral remote sensing data for forest biomass assessment. ISPRS Journal of Photogrammetry and Remote Sensing, 65(6), pp. 581-590.

Kurz, T.H., Buckley, S.J., Howell, J.A. and Schneider, D., 2008. Geological outcrop modelling and interpretation using ground based hyperspectral and laser scanning data fusion. International Archives of the Photogrammetry, Remote Sensing and Spatial Information Sciences, 37(B8), pp. 12291234.

Kurz, T.H., Buckley, S.J., Howell, J.A. and Schneider, D., 2011. Integration of panoramic hyperspectral imaging with terrestrial lidar data. Photogrammetric Record, 26(134), pp. 212-228.

Kurz, T.H., Dewit, J., Buckley, S.J., Thurmond, J.B., Hunt, D.W. and Swennen, R., 2012. Hyperspectral image analysis of different carbonate lithologies (limestone, karst and hydrothermal dolomites): the Pozalagua Quarry case study (Cantabria, North-west Spain). Sedimentology, 59(2), pp. 623-645.

Lichti, D.D., 2005. Spectral filtering and classification of terrestrial laser scanner point clouds. Photogrammetric Record, 20(111), pp. 218-240.

Luhmann, T., Robson, S., Kyle, S. and Harley, I., 2006. Close Range Photogrammetry: Principles, Methods and Applications. Whittles, Dunbeath, Caithness, Scotland. 510pp.

Nield, J.M., Wiggs, G.F. and Squirrell, R.S., 2011. Aeolian sand strip mobility and protodune development on a drying beach: examining surface moisture and surface roughness patterns measured by terrestrial laser scanning. Earth Surface Processes and Landforms, 36(2), pp. 273-278.

Norsk Elektro Optikk, 2012. HySpex hyperspectral imaging. http://www.hyspex.no/ (13 April, 2012).

Schneider, D. and Maas, H.-G., 2006. A geometric model for linear-array-based terrestrial panoramic cameras. Photogrammetric Record, 21(115), pp. 198-210.

van der Meer, F.D., de Jong, S.M., 2001. Imaging Spectrometry: Basic Principles and Prospective Applications, Kluwer Academic, Dordrecht, 403pp. 